\title{
Energy-Efficient Power Allocation for Fixed-Gain Amplify-and-Forward Relay Networks with Partial Channel State Information
}

\author{
Ammar Zafar, Student Member, IEEE, Redha M. Radaydeh, Member IEEE, Yunfei Chen, \\ Senior Member, IEEE, and Mohamed-Slim Alouini, Fellow, IEEE
}

\begin{abstract}
In this letter, energy-efficient transmission and power allocation for fixed-gain amplify-and-forward relay networks with partial channel state information (CSI) are studied. In the energy-efficiency problem, the total power consumed is minimized while keeping the signal-to-noise-ratio (SNR) above a certain threshold. In the dual problem of power allocation, the end-to-end SNR is maximized under individual and global power constraints. Closed-form expressions for the optimal source and relay powers and the Lagrangian multiplier are obtained. Numerical results show that the optimal power allocation with partial CSI provides comparable performance as optimal power allocation with full CSI at low SNR.

Index Terms-Amplify-and-Forward, cooperative communications, energy-efficiency, fixed-gain relays, optimal power allocation.
\end{abstract}

\section{INTRODUCTION}

Amplify-and-forward (AF) relaying is a popular cooperative relaying protocol which combats the detrimental effect of fading in wireless networks [1]. There are two main types of AF relays: 1) Variable-gain, 2) Fixed-gain. In variablegain relays, the relay gain is dependent on the instantaneous channel gain between the relay and the source and changes as the instantaneous channel gain changes. Hence, variablegain relays require knowledge of the instantaneous channel gain. In fixed-gain relays, the relay gain is constant and does not change with the changing channel gain. In the following fixed-gain AF relays are considered.

In a cooperative system, the energy available at the source and the relays is limited. Hence, it is crucial to utilize this energy as efficiently as possible. Furthermore, it is quite challenging to estimate the CSI of relay networks with a large number of relays. Hence, it is desirable to have power allocation schemes which require partial CSI. Power allocation for a three node system, source, destination and a single relay, to maximize the sum and product of the SNRs of the sourcedestination (S-D) and relay-destination (R-D) links requiring the knowledge of only the channel mean was studied in [2]. Optimal and near-optimal power allocation to maximize the end-to-end SNR for a three node system with fixed-gain AF relays which required knowledge of slowly varying CSI were studied in [3]. The optimal solution was obtained numerically, hence a closed-form near optimal solution was then proposed.

Different from [2], [3], a fixed-gain AF relaying network with multiple relays is considered in this letter. In addition to optimal power allocation, the problem of energy-efficiency, where the end-to-end SNR is kept above a threshold while the total power consumed is minimized, is also studied. It is assumed that the destination has complete CSI while the relays have complete knowledge of the channel gains of the R-D links and knowledge of only the channel statistics of the S-D and $\mathrm{S}-\mathrm{R}$ links. The channels gains are assumed to follow independent Generalized- $\mathcal{K}$ distributions [4]. Closed-form solutions are obtained using the Lagrangian multiplier method [5] for energy-efficient transmission and optimal power allocation.

\section{SySTEM MODEL}

Consider a system in which a source node transmits information to the destination node with the help of $m$ AF relays. The source and relays transmit on orthogonal channels. The received signals at the destination and the $i$ th relay from the source are

$$
y_{s d}=\sqrt{E_{s}} h_{s d} s+n_{s d} \quad y_{s i}=\sqrt{E_{s}} h_{s i} s+n_{s i}
$$

respectively, where $E_{s}$ is the energy used for transmission by the source node, $s$ is the zero mean unit energy transmitted symbol, $n_{s d}$ and $n_{s i}$ are $C N \sim\left(0, \sigma_{s d}^{2}\right)$ and $C N \sim\left(0, \sigma_{s i}^{2}\right)$ respectively, $h_{s d}$ and $h_{s i}$ are the unknown fading gains between the source and the destination, and between the source and the $i$ th relay, respectively. The fading gains are modeled as independent Generalized- $\mathcal{K}$ random variables. The Generalized- $\mathcal{K}$ distribution is a generalized distribution and contains the widely used Rayleigh, Nakagami-m and Rician distributions as special cases. The received signal at the destination from the $i$ th relay is

$$
y_{i d}=\sqrt{a_{i} E_{s} E_{i}} h_{s i} h_{i d} s+n_{i},
$$

where $E_{i}$ is the energy used for transmission by the $i$ th relay, $h_{i d}$ is the known fading gain between the $i$ th relay and the destination, $n_{i} \sim C N\left(0, \sigma_{i}^{2}\right)$ and $\sigma_{i}^{2}=a_{i} E_{i}\left|h_{i d}\right|^{2} \sigma_{s i}^{2}+\sigma_{i d}^{2}$, and $a_{i}$ is the amplification factor of the $i$ th relay. Assuming maximal ratio combining at the receiver, the end-to-end SNR is given by

$$
\gamma=E_{s}\left(\sum_{i=0}^{m} \alpha_{i}-\sum_{i=1}^{m} \frac{\alpha_{i} \zeta_{i}}{a_{i} E_{i} \beta_{i}+\zeta_{i}}\right),
$$

where $\alpha_{0}=\frac{\left|h_{s d}\right|^{2}}{\sigma_{s d}^{2}}, \alpha_{i}=\frac{\left|h_{s i}\right|^{2}}{\sigma_{s i}^{2}}, i=1, \ldots, m, \beta_{i}=\frac{\left|h_{i d}\right|^{2}}{\sigma_{i d}^{2}}$ and $\zeta_{i}=\frac{1}{\sigma_{s i}^{2}}$. As the values of $\alpha_{i} \mathrm{~s}$ are unknown at the relays, the SNR has to be averaged over them. As $h_{s d}$ and 
$h_{s i}$ are Generalized- $\mathcal{K}$ RVs, $\alpha_{i}$ follows the Gamma-Gamma distribution, given by [4]

$$
f_{\alpha_{i}}(x)=\frac{2 x^{\frac{k_{i}+l_{i}}{2}-1}}{\Gamma\left(k_{i}\right) \Gamma\left(l_{i}\right)}\left(\frac{k_{i} l_{i}}{\bar{\gamma}_{i}}\right)^{\frac{k_{i}+l_{i}}{2}} K_{k_{i}-l_{i}}\left[2 \sqrt{\frac{k_{i} l_{i} x}{\bar{\gamma}_{i}}}\right],
$$

where $k_{i} \geq 0, l_{i} \geq 0$ are the distribution shaping parameters associated with the two underlying Gamma random variables, $K_{v}($.$) is the modified Bessel function of the second kind$ and order $v$ [6, eqn (8.407.1)], $\Gamma($.$) is the Gamma function$ defined as $\Gamma(z)=\int_{0}^{\infty} e^{-t} t^{z-1} d t, \bar{\gamma}_{i}=\mathbb{E}\left[\alpha_{i}\right]$ and $\mathbb{E}[$.$] is$ the expectation operator. As $\alpha_{i} \mathrm{~s}$ are independent, their joint probability density function (pdf) is given by

$$
f_{\alpha_{0}, \alpha_{1} \ldots \alpha_{m}}\left(x_{0}, x_{1} \ldots x_{m}\right)=\prod_{i=0}^{m} f_{\alpha_{i}}\left(x_{i}\right),
$$

where $f_{\alpha_{i}}\left(x_{i}\right)$ is given in (4). Averaging (3) over (5) yields the SNR, $\gamma_{p c}$, for the system with partial knowledge of CSI

$$
\gamma_{p c}=E_{s}\left(\sum_{i=0}^{m} \bar{\gamma}_{i}-\sum_{i=1}^{m} \frac{\bar{\gamma}_{i} \zeta_{i}}{a_{i} E_{i} \beta_{i}+\zeta_{i}}\right) .
$$

Essentially, $\alpha_{i}$ in (3) has been replaced by $\bar{\gamma}_{i}$ in (6).

\section{ENERGY EFFICIENCY}

In the energy-efficiency problem, the total power consumed is minimized while maintaining $\gamma_{p c}$ above a predetermined threshold $\gamma^{t h}$. Assuming a unit symbol time, $E_{s}$ and $E_{i}$ can be thought of as source power and $i$ th relay power, respectively. The optimization problem is given by

$$
\begin{gathered}
\min \left(E_{s}+\sum_{i=1}^{m} E_{i}\right), \quad \text { subject to } \\
\gamma_{p c} \geq \gamma^{t h}, 0 \leq E_{s} \leq E_{s}^{\text {max }}, 0 \leq E_{i} \leq E_{i}^{\text {max }},
\end{gathered}
$$

where $E_{s}^{\max }$ and $E_{i}^{\max }$ specify the maximum power available at the source and the $i$ th relay, respectively. The objective function and the constraints are both convex functions of $E_{s}$ and $E_{i}$, except for $\gamma_{p c}$ which is concave on the domain of interest. Moreover, as the objective function and $\gamma_{p c}$ are monotonically increasing function of the powers, the optimal solution is achieved when $\gamma_{p c}=\gamma^{t h}$. As the other constraints are affine, it follows that Slater's condition [5] is satisfied. Therefore, the Lagrange multiplier method can be used to find the solution. Ignoring the individual constraints and forming the Lagrangian [5]

$\mathbb{L}=E_{s}+\sum_{i=1}^{m} E_{i}+\rho\left(\gamma^{t h}-E_{s} \sum_{i=0}^{m} \bar{\gamma}_{i}+\sum_{i=1}^{m} \frac{E_{s} \bar{\gamma}_{i} \zeta_{i}}{a_{i} E_{i} \beta_{i}+\zeta_{i}}\right)$,

where $\rho$ is the Lagrange multiplier. Solving the problem yields the solution

$$
E_{s}=\left(\frac{\rho\left(\sum_{j=1}^{m} \sqrt{\frac{\bar{\gamma}_{j} \zeta_{j}}{a_{j} \beta_{j}}}\right)^{2}}{\left(\rho \sum_{i=0}^{m} \bar{\gamma}_{i}-1\right)^{2}}\right)^{+}
$$

$$
\begin{gathered}
E_{j}=\left(\frac{\rho\left(\sum_{i=1}^{m} \sqrt{\frac{\bar{\gamma}_{i} \zeta_{i}}{a_{i} \beta_{i}}}\right)}{\left(\rho \sum_{i=0}^{m} \bar{\gamma}_{i}-1\right)} \sqrt{\frac{\bar{\gamma}_{j} \zeta_{j}}{a_{j} \beta_{j}}}-\frac{\zeta_{j}}{a_{j} \beta_{j}}\right)^{+} \\
\rho=\frac{\left(\sum_{j=1}^{m} \sqrt{\frac{\bar{\gamma}_{j} \zeta_{j}}{a_{j} \beta_{j}}}\right)}{\sum_{i=0}^{m} \bar{\gamma}_{i} \sqrt{\gamma^{t h}}}+\frac{1}{\sum_{i=0}^{m} \bar{\gamma}_{i}} .
\end{gathered}
$$

The total power consumed is a monotonically increasing function of $E_{s}$ and $E_{i}$. Therefore, the optimal power allocation after incorporating the individual constraints is

$$
\begin{gathered}
E_{s}=\left(\frac{\rho\left(\sum_{j=1}^{m} \sqrt{\frac{\bar{\gamma}_{j} \zeta_{j}}{a_{j} \beta_{j}}}\right)^{2}}{\left(\rho \sum_{i=0}^{m} \bar{\gamma}_{i}-1\right)^{2}}\right)_{0}^{E_{s}^{\max }} \\
E_{j}=\left(\frac{\rho\left(\sum_{i=1}^{m} \sqrt{\frac{\bar{\gamma}_{i} \zeta_{i}}{a_{i} \beta_{i}}}\right)}{\left(\rho \sum_{i=0}^{m} \bar{\gamma}_{i}-1\right)} \sqrt{\frac{\bar{\gamma}_{j} \zeta_{j}}{a_{j} \beta_{j}}}-\frac{\zeta_{j}}{a_{j} \beta_{j}}\right)_{0}^{E_{j}^{\max }} .
\end{gathered}
$$

The above can be viewed as a water-filling solution. Hence, the power is allocated in an iterative manner. Initially, the problem is solved without factoring in the individual constraints. Let $\mathbb{U}, \mathbb{W}$ and $\mathbb{V}$ be the sets of all powers which exceed their respective individual constraints, all powers below 0 and all powers which lie between the upper and lower constraints, respectively. The maximum power in $\mathbb{U}$ is set at its individual constraint and the process is repeated. If $\mathbb{U}$ is an empty set, i.e. no power exceeds its individual constraint, then the minimum power in $\mathbb{W}$ is set at 0 and the process is repeated. This iterative procedure is carried out until all the powers satisfy their individual constraints. However, in each iteration, the problem changes as the powers which are fixed are no longer variables of optimization. Thus, the objective function, the constraints and the closed-form solution changes. There can be two cases depending on the value of $E_{s}$. If $E_{s}$ is greater than $E_{s}^{\max }$, then it is set at $E_{s}^{\max }$, and all the $E_{i} \mathrm{~s}$ which belong to $\mathbb{U}$ are set to their respective peak constraint and $\gamma_{p c}$ is changed to

$\gamma_{p c}=E_{s}^{\max } \sum_{i \notin \mathbb{W}} \bar{\gamma}_{i}-\sum_{i \in \mathbb{V}} \frac{E_{s}^{\max } \bar{\gamma}_{i} \zeta_{i}}{a_{i} E_{i} \beta_{i}+\zeta_{i}}-\sum_{i \in \mathbb{U}} \frac{E_{s}^{\max } \bar{\gamma}_{i} \zeta_{i}}{a_{i} E_{i}^{\max } \beta_{i}+\zeta_{i}}$

Solving the optimization problem with the updated variables of optimization and $\gamma_{p c}$ gives the expression for $E_{j} \in \mathbb{V}$ [7]

$$
E_{j}=\left(\sqrt{\frac{\rho \bar{\gamma}_{j} \zeta_{j} E_{s}^{\max }}{a_{j} \beta_{j}}}-\frac{\zeta_{j}}{a_{j} \beta_{j}}\right)_{0}^{E_{j}^{\max }} .
$$

Putting (11) in the constraint $\gamma_{p c}=\gamma_{t h}$ gives $\rho$ as

$$
\rho=\frac{E_{s}^{\max }\left(\sum_{i \in \mathbb{V}} \sqrt{\frac{\bar{\gamma}_{i} \zeta_{i}}{a_{i} \beta_{i}}}\right)^{2}}{\left(E_{s}^{\max }\left(\sum_{i \notin \mathbb{W}} \bar{\gamma}_{i}-\sum_{i \in \mathbb{U}} \frac{\bar{\gamma}_{i} \zeta_{i}}{a_{i} E_{i}^{\max } \beta_{i}+\zeta_{i}}\right)-\gamma^{t h}\right)^{2}} .
$$


Similarly if $E_{s}$ lies in $\mathbb{V}$, then $\gamma_{p c}$ is given by

$$
\gamma_{p c}=E_{s} \sum_{i \notin \mathbb{W}} \bar{\gamma}_{i}-\sum_{i \in \mathbb{V}} \frac{E_{s} \bar{\gamma}_{i} \zeta_{i}}{a_{i} E_{i} \beta_{i}+\zeta_{i}}-\sum_{i \in \mathbb{U}} \frac{E_{s} \bar{\gamma}_{i} \zeta_{i}}{a_{i} E_{i}^{\text {max }} \beta_{i}+\zeta_{i}},
$$

Solving the updated optimization problem gives [7]

$$
\begin{aligned}
& E_{s}=\left(\frac{\rho\left(\sum_{j \in \mathbb{V}} \sqrt{\frac{\bar{\gamma}_{j} \zeta_{j}}{a_{j} \beta_{j}}}\right)^{2}}{\left(\rho \sum_{i \notin \mathbb{W}} \bar{\gamma}_{i}-\rho \sum_{i \in \mathbb{U}} \frac{\bar{\gamma}_{i} \zeta_{i}}{a_{i} E_{i}^{\max } \beta_{i}+\zeta_{i}}-1\right)^{2}}\right)_{0}^{E_{s}^{\max }} \\
& E_{j}=\left(\frac{\rho\left(\sum_{i \in \mathbb{V}} \sqrt{\frac{\bar{\gamma}_{i} \zeta_{i}}{a_{i} \beta_{i}}}\right) \sqrt{\frac{\bar{\gamma}_{j} \zeta_{j}}{a_{j} \beta_{j}}}}{\rho \sum_{i \notin \mathbb{W}} \bar{\gamma}_{i}-\rho \sum_{i \in \mathbb{U}} \frac{\bar{\gamma}_{i} \zeta_{i}}{a_{i} E_{i}^{\text {max }} \beta_{i}+\zeta_{i}}-1}-\frac{\zeta_{j}}{a_{j} \beta_{j}}\right)_{0}^{E_{j}^{\text {max }}}
\end{aligned}
$$

where

$$
\rho=\frac{\sqrt{\frac{\left(\sum_{j \in \mathbb{V}} \sqrt{\frac{\bar{\gamma}_{j} \zeta_{j}}{a_{j} \beta_{j}}}\right)^{2}}{\gamma^{t h}}}+1}{\sum_{i \notin \mathbb{W}} \bar{\gamma}_{i}-\sum_{i \in \mathbb{U}} \frac{\bar{\gamma}_{i} \zeta_{i}}{a_{i} E_{i}^{\text {max }} \beta_{i}+\zeta_{i}}} .
$$

\section{Optimal Power Allocation}

In this section, the dual problem of optimal power allocation is considered. Here, $\gamma_{p c}$ is maximized under the individual and global power constraints on the system. The optimization problem is given by

$$
\max E_{s}\left(\sum_{i=0}^{m} \bar{\gamma}_{i}-\sum_{i=1}^{m} \frac{\bar{\gamma}_{i} \zeta_{i}}{a_{i} E_{i} \beta_{i}+\zeta_{i}}\right) \text {, subject to }
$$

$0 \leq E_{s} \leq E_{s}^{\max }, 0 \leq E_{i} \leq E_{i}^{\max }, \quad\left(E_{s}+\sum_{i=1}^{m} E_{i}\right) \leq E_{t o t}$.

The objective function is concave on its domain and monotonically increasing function of $E_{s}$ and $E_{i}$ and the constraints are affine functions of $E_{s}$ and $E_{i}$. Therefore, Slater's condition is satisfied. Invoking the Lagrangian multiplier method, one can write the Lagrangian as

$$
\begin{aligned}
\mathbb{L}=E_{s} & \left(-\sum_{i=0}^{m} \bar{\gamma}_{i}+\sum_{i=1}^{m} \frac{\bar{\gamma}_{i} \zeta_{i}}{a_{i} E_{i} \beta_{i}+\zeta_{i}}\right)+ \\
& \delta\left(E_{s}+\sum_{i=1}^{m} E_{i}-E_{t o t}\right),
\end{aligned}
$$

where $\delta$ is the Lagrangian multiplier and the individual power constraints have been ignored. Solving the problem yields the solution

$$
E_{s}=\left(\frac{\delta\left(\sum_{i=1}^{m} \sqrt{\frac{\bar{\gamma}_{i} \zeta_{i}}{a_{i} \beta_{i}}}\right)^{2}}{\left(\sum_{i=0}^{m} \bar{\gamma}_{i}-\delta\right)^{2}}\right)^{+}
$$

$$
E_{j}=\left(\frac{\left(\sum_{i=1}^{m} \sqrt{\frac{\bar{\gamma}_{i} \zeta_{i}}{a_{i} \beta_{i}}}\right)}{\left(\sum_{i=0}^{m} \bar{\gamma}_{i}-\delta\right)} \sqrt{\frac{\bar{\gamma}_{j} \zeta_{j}}{a_{j} \beta_{j}}}-\frac{\zeta_{j}}{a_{j} \beta_{j}}\right)^{+}
$$

where

$$
\delta=\sum_{i=0}^{m} \bar{\gamma}_{i}-\left(\sum_{i=1}^{m} \sqrt{\frac{\bar{\gamma}_{i} \zeta_{i}}{a_{i} \beta_{i}}}\right) \sqrt{\frac{\left(\sum_{i=0}^{m} \bar{\gamma}_{i}\right)}{E_{t o t}+\sum_{j=1}^{m} \frac{\zeta_{j}}{a_{j} \beta_{j}}}} .
$$

As the objective function is a monotonically increasing function of the source and relays powers, the optimal power allocation after incorporating the individual power constraints is given by

$$
\begin{gathered}
E_{s}=\left(\frac{\delta\left(\sum_{i=1}^{m} \sqrt{\frac{\bar{\gamma}_{i} \zeta_{i}}{a_{i} \beta_{i}}}\right)^{2}}{\left(\sum_{i=0}^{m} \bar{\gamma}_{i}-\delta\right)^{2}}\right)_{0}^{E_{s}^{\max }} \\
E_{j}=\left(\frac{\left(\sum_{i=1}^{m} \sqrt{\frac{\bar{\gamma}_{i} \zeta_{i}}{a_{i} \beta_{i}}}\right)}{\left(\sum_{i=0}^{m} \bar{\gamma}_{i}-\delta\right)} \sqrt{\frac{\bar{\gamma}_{j} \zeta_{j}}{a_{j} \beta_{j}}}-\frac{\zeta_{j}}{a_{j} \beta_{j}}\right)_{0}^{E_{j}^{\max }} .
\end{gathered}
$$

As was the case in the previous section, the optimal power allocation follows a water-filling solution. Hence, the power is allocated iteratively and follows the same algorithm as described in the previous section. Employing the same notation as before, if $E_{s}$ lies in $\mathbb{U}$, then $\gamma_{p c}$ is given by (10) and the total power constraint is updated to

$$
E_{t o t}=E_{t o t}-E_{s}^{\max }-\sum_{i \in \mathbb{U}} E_{i}^{\max } .
$$

Solving the problem with the updated objective function and constraints gives the new expression for $E_{j} \in \mathbb{V}$ as [7]

$$
E_{j}=\left(\sqrt{\frac{E_{s}^{\max } \bar{\gamma}_{j} \zeta_{j}}{\delta a_{j} \beta_{j}}}-\frac{\zeta_{j}}{a_{j} \beta_{j}}\right)_{0}^{E_{j}^{\max }}
$$

where the Lagrangian multiplier is given by

$$
\delta=\frac{E_{s}^{\max }\left(\sum_{j \in \mathbb{V}} \sqrt{\frac{\bar{\gamma}_{j} \zeta_{j}}{a_{j} \beta_{j}}}\right)^{2}}{\left(E_{t o t}-E_{s}^{\max }-\sum_{i \in \mathbb{U}} E_{i}^{\max }+\sum_{j \in \mathbb{V}} \frac{\zeta_{j}}{a_{j} \beta_{j}}\right)^{2}},
$$

Similarly, if $E_{s}$ lies in $\mathbb{V}$, then the new expressions can be obtained as [7]

$$
\begin{aligned}
& E_{s}=\left(\frac{\delta\left(\sum_{i \in \mathbb{V}} \sqrt{\frac{\bar{\gamma}_{i} \zeta_{i}}{a_{i} \beta_{i}}}\right)^{2}}{\left(\sum_{i \notin \mathbb{W}} \bar{\gamma}_{i}-\delta-\sum_{i \in \mathbb{U}} \frac{\bar{\gamma}_{i} \zeta_{i}}{a_{i} E_{i}^{\max } \beta_{i}+\zeta_{i}}\right)^{2}}\right)_{0}^{E_{s}^{\max }} \\
& E_{j}=\left(\frac{\left(\sum_{i \in \mathbb{V}} \sqrt{\frac{\bar{\gamma}_{i} \zeta_{i}}{a_{i} \beta_{i}}}\right) \sqrt{\frac{\bar{\gamma}_{j} \zeta_{j}}{a_{j} \beta_{j}}}}{\sum_{i \notin \mathbb{W}} \bar{\gamma}_{i}-\delta-\sum_{i \in \mathbb{U}} \frac{\bar{\gamma}_{i} \zeta_{i}}{a_{i} E_{i}^{\text {max }} \beta_{i}+\zeta_{i}}}-\frac{\zeta_{j}}{a_{j} \beta_{j}}\right)_{0}^{E_{j}^{m a}}
\end{aligned}
$$




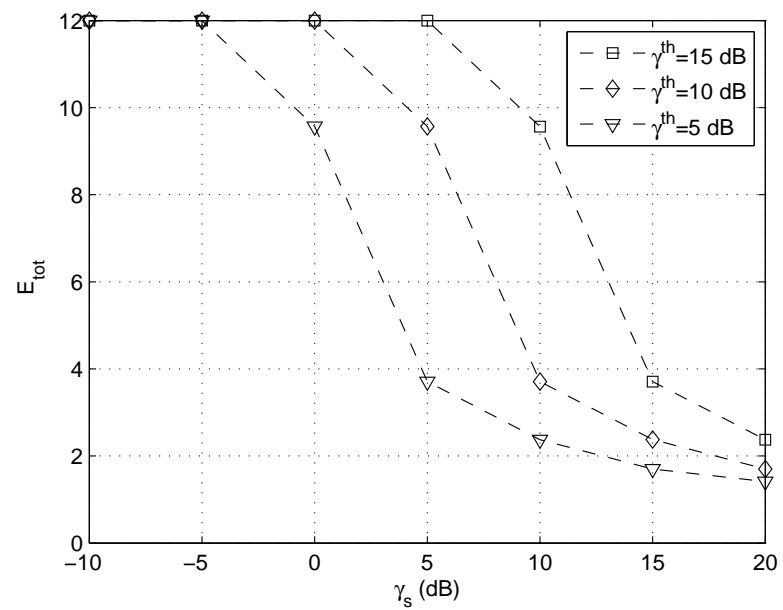

Fig. 1: Total energy consumed as a function of $\gamma_{s}$ for $m=3$.

where

$$
\begin{gathered}
\delta=\sum_{i \notin \mathbb{W}} \bar{\gamma}_{i}-\sum_{i \in \mathbb{U}} \frac{\bar{\gamma}_{i} \zeta_{i}}{a_{i} E_{i}^{\text {max }} \beta_{i}+\zeta_{i}}-\sum_{i \in \mathbb{V}} \sqrt{\frac{\bar{\gamma}_{i} \zeta_{i}}{a_{i} \beta_{i}}} \times \\
\sqrt{\frac{\sum_{i \notin \mathbb{W}} \bar{\gamma}_{i}-\sum_{i \in \mathbb{U}} \frac{\bar{\gamma}_{i} \zeta_{i}}{a_{i} E_{i}^{\text {max }} \beta_{i}+\zeta_{i}}}{E_{\text {tot }}-\sum_{i \in U} E_{i}^{\text {max }}+\sum_{j \in \mathbb{V}} \frac{\zeta_{j}}{a_{j} \beta_{j}}}}
\end{gathered}
$$

\section{NUMERICAL RESUlTS AND DISCUSSION}

Numerical results are discussed in this section. $E_{s}^{\max }$ and $E_{i}^{\max }$ are taken to be 3 . All $\bar{\gamma}_{i} \mathrm{~s}$ are taken to be 0.5 . For optimal power allocation, $E_{t o t}$ is set at 9 . All noise variances set equal as $\sigma_{s d}^{2}=\sigma_{s i}^{2}=\sigma_{i d}^{2}=\sigma^{2}$. The relay gain, $a_{i}$, of each relay is taken to be 1 . The results are averaged over one million channel realizations. For the energy-efficiency problem, depending on the system and channel conditions, it might not be possible to achieve the desired $\gamma_{t h}$. In such instances, the source and all the relays will transmit at their individual constraint to achieve maximum possible $\gamma_{p c}$.

Figure 1 shows the optimized $E_{t o t}$, where $E_{t o t}=E_{s}+$ $\sum_{i=1}^{m} E_{i}$, as a function of $\gamma_{s}$, where $\gamma_{s}=\frac{1}{\sigma^{2}}$ for a cooperative system with three relays to examine the effect of the proposed energy-efficiency solution. For low values of $\gamma_{s}$ the total energy consumed, $E_{t o t}$, is constant at the peak of 12 for all three values of $\gamma^{\text {th }}$. This is due to the fact that because of the system constraints and large amount of noise in the system, the desired SNR threshold cannot be achieved and the source and the relays all transmit at their respective individual constraint which in the numerical example is 3 . Hence, the total energy consumed is 12 . Moreover, with increasing $\gamma^{\text {th }}$, the range of values of $\gamma_{s}$ for which the system consumes all available power increases as more power is needed to achieve a higher $\gamma^{t h}$. For high values of $\gamma_{s}, E_{t o t}$ decreases as there is less noise in the system and less transmit power is required to achieve the desired $\gamma^{\text {th }}$.

Figure 2 shows the symbol error rate performance of

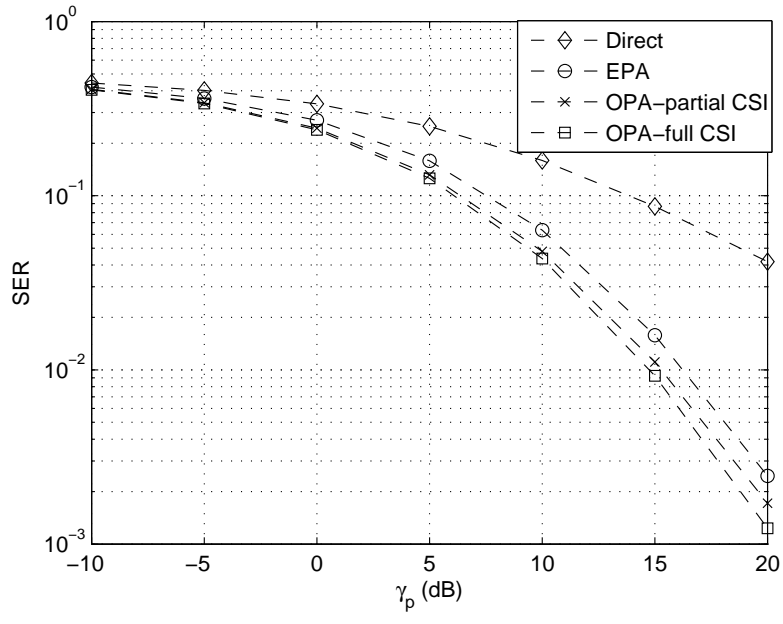

Fig. 2: Comparison of SER performance.

optimal power allocation (OPA) with full CSI, OPA with partial CSI, equal power allocation (EPA) and the direct link with respect to $\gamma_{p}$, where $\gamma_{p}=\frac{E_{t o t}}{\sigma^{2}}$, for binary phase shift keying (BPSK) to examine the effect of the proposed power allocation scheme. OPA with partial CSI provides comparable performance to OPA with full CSI at low $\gamma_{p}$. However, the difference in performance increases with increasing $\gamma_{p}$. At an error rate of $10^{-2}$. the performance difference between OPAfull CSI and OPA-partial CSI is about $0.5 \mathrm{~dB}$, however, the latter requires less complexity. At the same error rate the gain of OPA-partial CSI over EPA is $1 \mathrm{~dB}$.

\section{CONCLUSiOnS}

In this paper, closed-form expressions for the source and relay powers have been derived for the dual problems of minimizing total power consumed while maintaining the SNR above a threshold and optimal power allocation to maximize SNR. It has been shown that for both problems, the power allocation follows a water-filling solution. Numerical results have shown that optimal power allocation outperforms the EPA scheme.

\section{REFERENCES}

[1] J. Laneman, D. Tse, and G. Wornell, "Cooperative diversity in wireless networks: Efficient protocols and outage behavior," IEEE Transactions on Information Theory, vol. 50, no. 12, pp. 3062-3080, Dec. 2004.

[2] X. Deng and A. Haimovich, "Power allocation for cooperative relaying in wireless networks," IEEE Communications Letters, vol. 9, no. 11, pp. 994-996, 2005.

[3] T. Riihonen and R. Wichman, "Power allocation for a single-frequency fixed-gain relay network," in Proceedings IEEE 18th International Symposium on Personal, Indoor and Mobile Radio Communications (PIMRC), Athens, Greece, Sep. 2007, pp. 1-5.

[4] A. Laourine, M.-S. Alouini, S. Affes, and A. Stéphenne, "On the capacity of generalized- $\mathcal{K}$ fading channels," IEEE Transactions on Wireless Communications, vol. 7, no. 7, pp. 2441-2445, Jul. 2007.

[5] S. Boyd and L. Vandenberghe, Convex Optimization. New York, USA: Cambridge University Press, 2009.

[6] I. S. Gradshteyn and I. M. Ryzhik, Table of Integrals, Series, and Products, 7th ed. New York, USA: Academic Press, 2007.

[7] A. Zafar, R. M. Radaydeh, Y. Chen, and M.-S. Alouini, "Energy-efficient power allocation for fixed-gain amplify-and-forward relay networks with partial channel state information," King Abdullah Univeristy of Science and Technology (KAUST), Tech. Rep., Jun. 2012, available at http://hdl.handle.net/10754/230312. 